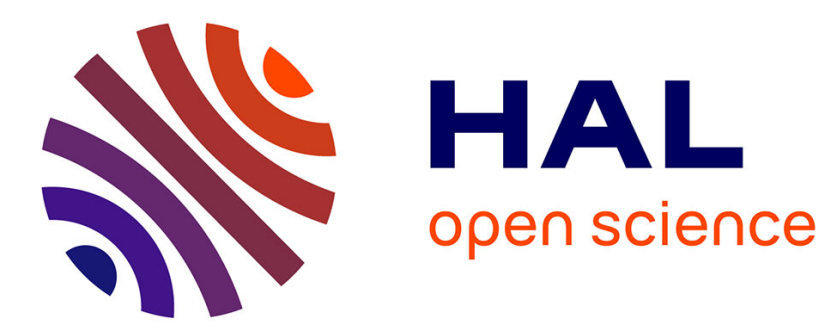

\title{
Mobilité quotidienne des citadins à faibles ressources. Les enseignements de Ouagadougou
}

\author{
Lourdes Diaz Olvera, Didier Plat, Pascal Pochet
}

\section{To cite this version:}

Lourdes Diaz Olvera, Didier Plat, Pascal Pochet. Mobilité quotidienne des citadins à faibles ressources. Les enseignements de Ouagadougou. Revue Tiers Monde, 1999, XL (160), pp.829-848. halshs00958202

\section{HAL Id: halshs-00958202 \\ https://shs.hal.science/halshs-00958202}

Submitted on 11 Mar 2014

HAL is a multi-disciplinary open access archive for the deposit and dissemination of scientific research documents, whether they are published or not. The documents may come from teaching and research institutions in France or abroad, or from public or private research centers.
L'archive ouverte pluridisciplinaire HAL, est destinée au dépôt et à la diffusion de documents scientifiques de niveau recherche, publiés ou non, émanant des établissements d'enseignement et de recherche français ou étrangers, des laboratoires publics ou privés. 


\title{
Mobilité quotidienne des citadins à faibles ressources. Les enseignements de Ouagadougou
}

\author{
Lourdes Diaz Olvera, Didier Plat, Pascal Pochet
}

\author{
Laboratoire d'Economie des Transports \\ ENTPE - Université Lumière Lyon 2 - CNRS \\ Ecole Nationale des Travaux Publics de l'Etat \\ rue Maurice Audin \\ 69518 Vaulx-en-Velin cedex \\ lourdes.diaz-olvera@entpe.fr; didier.plat@entpe.fr; pascal.pochet@entpe.fr
}

référence :

Diaz Olvera L., Plat D., Pochet P. (1999), Mobilité quotidienne des citadins à faibles ressources. Les enseignements de Ouagadougou, Revue Tiers Monde, Vol. XL, n¹60, pp. 829-848. 


\section{Mobilité quotidienne des citadins à faibles ressources. Les enseignements de Ouagadougou}

\section{Résumé}

La mobilité quotidienne s'avère problématique pour la majorité des habitants des grandes villes africaines (coût élevé, longs déplacements à pied...). Pour étudier les conséquences du manque de moyens sur les conditions de déplacement en milieu urbain, nous utilisons des données issues d'une enquête-ménages réalisée à Ouagadougou, ville où l'usage des deuxroues pallie les carences du système de transport. Les résultats font apparaître la grande variété des besoins et des pratiques concernant la mobilité quotidienne des différents groupes de citadins démunis, selon le rôle et la position dans le ménage notamment. Le coût élevé des véhicules motorisés réserve leur usage aux individus effectivement ou potentiellement productifs, ce qui laisse de côté une large part de la population, dont les besoins de transport sont pourtant loin d'être négligeables.

\section{Daily mobility of underprivileged urban dwellers. Evidence from Ouagadougou}

\section{Abstract}

Urban daily mobility brings about many problems for a large section of the population in SubSaharan African cities (high costs, long foot trips...). To study the consequences of lack of means on daily travel behaviour, we use data obtained from a household survey in Ouagadougou, where the lack of public transport is compensated by the use of two-wheelers. The analysis shows a wide spectrum of mobility requirements and practices amongst groups of underprivileged city-dwellers, according to social role and position within the household. Due to their high operating costs, private motorised vehicles are reserved to present-day or future bread winners of the households. Thus, a large part of the population is left behind due to this segregation. Nevertheless, they have considerable transport needs. 
Les populations urbaines d'Afrique subsaharienne se caractérisent par leur forte croissance numérique et, pour la grande majorité d'entre elles, par de faibles revenus. Du fait de l'étalement des villes et de l'allongement des distances de déplacement, la mobilité quotidienne s'avère problématique pour une large part des citadins. La marche à pied demeure généralement le mode de transport le plus utilisé, les longs déplacements à pied étant le lot quotidien des personnes démunies, comme cela a pu être mesuré à Bamako1. Peu développés, les moyens de transports motorisés, individuels ou publics, ont aussi l'inconvénient d'être onéreux. Si les données précises sont rares en ce domaine, on sait tout de même que les dépenses des ménages pour le transport peuvent atteindre des niveaux élevés (17\% des dépenses des ménages à Yaoundé, $23 \%$ du revenu individuel à Lagos pour les utilisateurs des transports collectifs) $)^{2}$, et s'avèrent particulièrement difficiles à assumer pour les plus pauvres. L'usage des transports collectifs est alors limité aux déplacements les plus indispensables à l'activité économique, comme l'ont montré des enquêtes auprès de petites commerçantes d'Accra ${ }^{3}$, ou d'actifs pauvres de Dakar4. La mobilité quotidienne de ces actifs pauvres apparaît très dépendante de la «conjoncture » (revenus de la journée ou de la semaine, opportunités de déplacement), tout en participant de stratégies d'amélioration des revenus.

Pourtant, les pauvres ne sont souvent, au mieux, que les bénéficiaires indirects des infrastructures de transport, la construction et l'entretien des voiries urbaines favorisant en premier lieu la mobilité quotidienne des personnes possédant une voiture, soit la minorité aux revenus élevés ${ }^{5}$. En effet, dans la plupart des capitales africaines, les taux de motorisation sont faibles et l'organisation des transports publics connaît de nombreux dysfonctionnements. Les grands réseaux de transport collectif ont bien du mal à se pérenniser; les entreprises artisanales (minibus, taxis collectifs) qui les concurrencent sont, elles aussi, confrontées à la contraction de la demande solvable depuis le début des années quatre-vingt. A ces problèmes généraux s'ajoute la difficulté de desservir les quartiers périphériques généralement non lotis et difficilement accessibles ${ }^{6}$.

\footnotetext{
1 Pochet et al., 1995.

2 Ngabmen, 1997 ; Sethi et Bhandari, 1996.

${ }^{3}$ Grieco et al., 1996.

4 Godard, 1997.

5 Diaz Olvera et Plat, 1997.

6 Godard et Teurnier, 1992.
} 
Dans une optique de développement, il paraît pourtant essentiel de faciliter les conditions dans lesquelles s'effectue la mobilité quotidienne des pauvres. Il en va en premier lieu de l'accès à l'emploi, vital lorsque les revenus sont bas. Mais il en va aussi de l'usage des équipements et services de base, tels que la santé et l'éducation7, comme de l'entretien des solidarités communautaires $^{8}$. Il en va enfin de l'amélioration des conditions de vie par la diminution de la pénibilité des déplacements urbains. L'inaccessibilité qualitative et quantitative au transport, qui prévaut dans bien des cas, peut neutraliser les résultats attendus dans d'autres secteurs.

Améliorer les conditions de transport des citadins démunis nécessite au préalable de mieux connaître quelles sont leurs pratiques de déplacements et leurs besoins en ce domaine. Dans cette optique, nous présentons ici une analyse de données recueillies lors d'une enquête quantitative auprès des habitants de Ouagadougou, après avoir brièvement précisé les spécificités du contexte urbain ouagalais, la méthodologie de l'enquête et la façon dont nous appréhendons les différentes situations individuelles de pauvreté.

\section{LE CONTEXTE OUAGALAIS : OMNIPRESENCE DES DEUX-ROUES MOTORISES, ROLE TRES MARGINAL DES TRANSPORTS COLLECTIFS}

La population de Ouagadougou est estimée aux environs de 800000 habitants. La période « révolutionnaire » (1983-1987) a donné lieu à un ensemble de mesures qui ont marqué le développement et l'organisation de la ville ${ }^{9}$. Parmi ces mesures, la redistribution des pouvoirs locaux, un nouveau découpage administratif et spatial de la ville et des programmes de lotissement dans les quartiers «spontanés» ont influencé plus ou moins directement les conditions de la mobilité quotidienne, en bouleversant l'organisation de l'espace urbain et les modes de vie des Ouagalais. Malgré cette politique volontariste d'aménagement urbain, des zones d'habitat non loti se sont développées au fil du temps dans la périphérie de la capitale burkinabé.

\footnotetext{
${ }^{7}$ Gannon et Liu, 1997.

${ }^{8}$ Marie $(1995,1997)$ montre qu'en période de crise et d'appauvrissement des populations urbaines, ces réseaux sont encore plus indispensables, mais aussi plus difficiles à activer. Leur efficacité est toutefois relative, tant richesse économique et richesse relationnelle vont de pair (Vuarin, 1997). Les conséquences en matière de mobilité quotidienne apparaissent clairement dans la biographie de Sali, dakaroise pauvre d'une cinquantaine d'années, que retrace Werner (1997). Ce récit de vie illustre bien les difficultés au jour le jour de l'entretien d'un capital relationnel, de sa conversion en capital économique et leur traduction très concrète en nécessités impérieuses de «déplacements incessants » qui valent à Sali le surnom de «marcheuse » (p. 383).

9 Jaglin et al., 1992 ; Jaglin, 1995.
} 
Contrairement à la majorité des capitales africaines, les transports collectifs sont apparus très tardivement et ne tiennent pas une place déterminante à Ouagadougou. La création de la première entreprise publique de transport collectif urbain, la "X9", ne date que de 1984. Dans un environnement très favorable, dépourvu de toute concurrence, l'offre de transport assurée par la X9 a été satisfaisante les premières années, pour se dégrader ensuite très nettement. Actuellement, les transports collectifs ont une place tout à fait minoritaire dans la répartition modale et les Ouagalais satisfont leurs besoins de transport soit en se déplaçant à pied, soit en se tournant très largement vers les modes individuels, notamment les deux-roues motorisés, dont l'apparition date des années soixante-dix. Ainsi, en 1992, pour 100 ménages on compte 150 cycles motorisés, 79 bicyclettes et 22 voitures, la taille moyenne des ménages étant légèrement inférieure à 8 personnes. L'enquête-ménages met en évidence des niveaux de mobilité élevés à Ouagadougou, de l'ordre de quatre déplacements quotidiens en moyenne, dont 2,2 déplacements utilisant un mode mécanisé (hors marche à pied), ce qui représente des taux supérieurs de plus de moitié à ceux d'autres capitales africaines. La large diffusion des deux-roues motorisés dans la population et le retour fréquent au domicile à la mi-journée expliquent cette mobilité élevée ${ }^{10}$.

\section{UNE ENQUETE AUPRES DES MENAGES}

Une enquête-ménages réalisée à Ouagadougou en février 1992 (soit avant la dévaluation du franc CFA) constitue le support empirique pour appréhender la mobilité quotidienne et les conditions de vie des citadins ${ }^{11}$. Le ménage intègre l'ensemble des personnes vivant sous le même toit et partageant le même feu, qu'elles soient ou non apparentées. Sa structure peut se révéler complexe et sa taille élevée. Le questionnaire permet de recueillir trois niveaux d'informations :

- des données générales sur le ménage : composition socio-démographique, caractéristiques de la parcelle et du logement, véhicules possédés ;

- des renseignements concernant chaque individu de plus de 13 ans : caractéristiques socioéconomiques, revenus, accès aux modes de transport, dépenses transport ;

\footnotetext{
10 Diaz Olvera et al., 1996.

11 Pour des résultats généraux sur la mobilité quotidienne issus de cette enquête, voir Diaz Olvera et Plat, 1994.
} 
- une description des déplacements effectués la veille du jour de l'enquête par chaque individu interrogé : motif, heures de départ et d'arrivée, mode de transport utilisé, lieux d'origine et de destination.

L'enquête a été réalisée dans 10 secteurs différents de la ville, de manière à obtenir une image aussi fidèle que possible des caractéristiques sociales et de la mobilité quotidienne des habitants de Ouagadougou. Les 3682 individus de plus de 13 ans enquêtés dans 753 ménages ont effectué 13659 déplacements urbains la veille du jour de l'enquête. La mobilité est évaluée par le nombre de déplacements par personne, que l'on peut décomposer par mode de transport ou encore par motif.

\section{LE POIDS DU TRANSPORT DANS LE REVENU DES MENAGES}

L'enquête nous renseigne sur les revenus gagnés par chaque individu de plus de 13 ans, sur ses dépenses d'usage (carburant, entretien et réparations, assurance et vignette) pour chaque véhicule possédé par le ménage (bicyclettes, deux-roues motorisés, voitures particulières) et enfin sur ses dépenses en transports collectifs. Pour chaque ménage, la somme des revenus et des dépenses déclarés par chacun des membres permet de connaître la part des dépenses de transport dans le revenu du foyer.

Le revenu total des ménages est estimé dans l'enquête à 119200 FCFA ${ }^{12}$ par mois en moyenne et la dépense mensuelle moyenne d'un ménage pour son transport à 23600 FCFA, soit 19,8\% des revenus ${ }^{13}$. Cette part dépend bien évidemment de possibilités d'accès aux moyens de transport individuel très variables selon le revenu, du fait de coûts d'usage fortement hiérarchisés entre modes. La voiture, dont le coût moyen mensuel s'élève à 37000 FCFA, demeure le privilège du cinquième des foyers les plus riches (tableau 1). L'usage d'un deuxroues motorisé occasionne des dépenses de l'ordre de 9400 FCFA mensuels, montant à comparer au salaire minimum, 22000 FCFA. Ce coût, loin d'être négligeable compte tenu du niveau de vie des Ouagalais, est aussi trois fois plus élevé que les dépenses moyennes pour les

\footnotetext{
12 Soit, au taux de change de l'époque, $2384 \mathrm{~F}$ français ( 1 FCFA = 2 centimes).

${ }^{13}$ La part des dépenses de transport serait plus élevée si l'on intégrait les coûts d'acquisition des véhicules. Bien que l'on dispose dans l'enquête des prix et des dates d'achat des véhicules, il est plus difficile de ramener les coûts d'achat à une moyenne de dépenses mensuelles, car on ne connaît ni la durée de vie des véhicules, ni la valeur de revente. Cependant, pour donner un ordre de grandeur des dépenses mensuelles liées à l'acquisition des véhicules, avec l'hypothèse d'une durée de vie de 10 ans, et d'une valeur de revente nulle, ce coût d'acquisition moyen par ménage peut être évalué à 7200 FCFA par mois (soit $6 \%$ en moyenne du revenu des ménages).
} 
transports collectifs déclarées par les usagers réguliers de ce mode (mais il permet des niveaux de mobilité motorisée bien supérieurs). Les ménages des 2ème, 3ème et 4ème quintiles, qui n'ont généralement pas accès à la voiture, disposent par contre de revenus suffisants pour posséder un ou plusieurs deux-roues motorisés. Enfin, la possession au mieux de bicyclette(s), véhicule dont le coût d'utilisation mensuel est inférieur à 1000 FCFA, se concentre dans le premier quintile, même si parmi les plus pauvres aussi, l'accès au deux-roues moteur constitue le cas le plus fréquent.

Tableau 1 : Equipement des ménages en moyens de transport, par quintile (\%)

\begin{tabular}{lcccccc}
\hline & $\begin{array}{c}\text { 1er } \\
\text { quintile }\end{array}$ & $\begin{array}{r}\text { 2ème } \\
\text { quintile }\end{array}$ & $\begin{array}{c}\text { 3ème } \\
\text { quintile }\end{array}$ & $\begin{array}{c}\text { 4ème } \\
\text { quintile }\end{array}$ & $\begin{array}{c}\text { 5ème } \\
\text { quintile }\end{array}$ & Moyenne \\
\hline Aucun véhicule ou bicyclette(s) & 42 & 14 & 7 & 5 & 0 & 13 \\
Deux-roues moteur* & 57 & 81 & 85 & 84 & 42 & 69 \\
Voiture(s)** & 1 & 5 & 8 & 11 & 58 & 18 \\
\hline Ensemble & 100 & 100 & 100 & 100 & 100 & 100 \\
\hline
\end{tabular}

* catégorie pouvant disposer aussi, éventuellement, de bicyclette(s).

** catégorie pouvant disposer aussi, éventuellement, de deux-roues moteur et de bicyclette(s).

La structure des dépenses par mode reflète bien la prédominance des deux-roues motorisés dans le système de transport ouagalais. Mobylettes et motos représentent $61 \%$ des dépenses totales (alors que les deux-roues motorisés assurent les deux tiers des déplacements mécanisés), la voiture, $29 \%$ (mais $11 \%$ des déplacements mécanisés), les transports en commun, $8 \%$ (pour $5 \%$ ) et la bicyclette, $2 \%$ seulement (pour $17 \%$ ). La part du revenu consacrée au transport décroît un peu lorsque le revenu augmente, confirmant en cela le fait que les dépenses pour les transports pèsent particulièrement lourd dans le budget des ménages les plus pauvres (tableau 2). 
Tableau 2 : Quintiles de revenu et dépenses de transport des ménages (FCFA et \%)

\begin{tabular}{lccc}
\hline & $\begin{array}{c}\text { Revenu } \\
\text { moyen }\end{array}$ & $\begin{array}{c}\text { Dépenses } \\
\text { moyennes }\end{array}$ & $\begin{array}{c}\text { \% du revenu } \\
\text { consacré au } \\
\text { transport }\end{array}$ \\
\hline 1er quintile <40 000 FCFA & 26800 & 6700 & 24,9 \\
2ème quintile [40 000-73 000[ & 56200 & 13800 & 24,5 \\
3ème quintile [73 000-108 000[ & 89300 & 18600 & 20,9 \\
4ème quintile [108 000-180 000[ & 138700 & 25900 & 18,7 \\
5ème quintile >180 000 & 303400 & 55800 & 18,4 \\
\hline Ensemble & 119200 & 23600 & 19,8 \\
\hline
\end{tabular}

Ces dépenses de transport élevées s'expliquent en partie par la diffusion importante du deuxroues moteur au sein de la population ouagalaise. Au sein des deux premiers quintiles, un ménage sur trois dépense plus du quart de son revenu en transport et près de $90 \%$ des dépenses sont alors affectées aux deux-roues motorisés. Cette proportion n'est plus que d'un sur cinq dans les 3ème et 4ème quintiles. A l'inverse, au sein du premier quintile, le tiers des ménages dépensent moins de $10 \%$ de leurs revenus en transport. Il s'agit des foyers trop pauvres pour acquérir une mobylette, et dont les membres se déplacent presque exclusivement à pied ou à vélo et rarement en transports collectifs. En comparaison, cette part n'est plus que de $11 \%$ dans le 2ème quintile. Cette analyse des écarts entre ménages, si elle montre des différentiels notables liés au revenu, ne permet toutefois pas d'appréhender les comportements de mobilité et doit être prolongée au niveau individuel.

\section{IDENTIFICATION DES INDIVIDUS A FAIBLES RESSOURCES}

En effet, dans des ménages inégalement dotés en moyens de transports, les véhicules sont bien insuffisants en nombre pour satisfaire les besoins de tous les membres. Aux disparités entre ménages s'ajoutent donc des inégalités d'accès aux véhicules au sein même des foyers, qui rendent nécessaire l'étude simultanée des situations socio-économiques individuelles au sein des ménages et leur impact sur les conditions de déplacements. Il s'agit donc moins ici de déterminer des populations pauvres au sens habituel du terme (fixation à partir des revenus des ménages d'une ligne de pauvreté séparant pauvres et non-pauvres) ${ }^{14}$ que de repérer des

\footnotetext{
14 Voir notamment Banque Mondiale (1990) et, pour une revue critique des mesures de la pauvreté, Salama et Valier (1995).
} 
situations dans lesquelles les individus sont susceptibles de souffrir d'un «manque de moyens » concernant non seulement les revenus, mais aussi l'absence d'autonomie au sein du ménage, la précarité professionnelle et résidentielle, les difficultés d'entretien des réseaux sociaux... ${ }^{15}$ Notre hypothèse est que, pour expliquer les différences de mobilité, les ressources individuelles et la place des individus dans le ménage sont plus déterminantes que les revenus du ménage.

Cette démarche n'est certes pas la plus courante : généralement est posée l'hypothèse d'une mise en commun des revenus de tous les membres du ménage. L'analyse se fait alors en évaluant les revenus et les dépenses moyens pour chaque membre du ménage, comme étant la somme des revenus ou des dépenses de tous les membres rapportée à la taille pondérée du ménage. Ce calcul permet d'intégrer les éventuels transferts entre membres du ménage. Cette procédure, tout à fait valide dans le cas par exemple de dépenses alimentaires, paraît insuffisante dans le domaine des déplacements quotidiens, où, comme nous allons le voir, les transferts semblent à la fois très limités (rareté et situation économique obligent) et très sélectifs quant aux destinataires (position dans le ménage oblige). C'est pourquoi, pour mettre en évidence ces inégalités de ressources entre ménages, mais aussi au sein des ménages, nous avons adopté une démarche en deux temps.

Dans un premier temps, nous repérons deux niveaux distincts de ressources: celles de l'individu et celles de son ménage. Nous cherchons ici à identifier, d'une part, des individus (et des ménages) démunis (ou à faibles ressources) et, d'autre part, des individus (et des ménages) aisés. Les ressources du ménage sont approchées à partir des caractéristiques socioéconomiques de son chef $^{16}$, tandis que les ressources individuelles sont définies en fonction des mêmes caractéristiques socio-économiques, mais cette fois-ci propres à chaque individu. Afin de disposer d'indicateurs plus stables dans le temps que le simple revenu, ces ressources sont évaluées à partir de trois variables : l'activité professionnelle (profession et secteur d'activité ) et, lorsque celle-ci n'est pas suffisante pour trancher, le type d'habitat et le niveau d'étude. Nous avons classé d'emblée parmi les individus aisés, les employeurs et les cadres moyens ou supérieurs du secteur privé ou de l'administration, et parmi les individus démunis,

\footnotetext{
15 Marie (1995).

${ }^{16}$ Le choix de l'estimation des ressources du ménage en fonction de la situation économique du chef repose sur le fait qu'il est dans la majorité des cas le principal soutien économique du foyer dans les villes d'Afrique de l'Ouest (Lachaud, 1995). Cette caractéristique se vérifie dans notre enquête : $61 \%$ des revenus des foyers proviennent du chef, lequel a le revenu le plus élevé de tous les actifs du foyer dans $83 \%$ des cas.
} 
les inactifs et chômeurs, les scolaires et les étudiants, de même que différents types d'actifs aux occupations peu rémunératrices et/ou d'un statut précaire (agriculteurs, artisans travaillant en indépendant ou employés, personnes vivant de petits métiers). Les autres catégories (employés ou ouvriers dans le commerce ou dans le secteur public, commerçants indépendants) sont plus hétérogènes. C'est pourquoi l'affectation s'est faite sur le type d'habitat (villa, logement en dur ou semi-dur / traditionnel en banco) et le niveau d'éducation (secondaire ou supérieur / sans ou primaire).

La combinaison de ces deux niveaux de ressources permet d'identifier quatre catégories: individus démunis appartenant à un ménage démuni, individus démunis dans un ménage aisé, individus aisés appartenant à un ménage démuni, individus aisés dans un ménage aisé. Les individus démunis sont largement dominants, puisque, selon la définition retenue, leur part dans la population est très élevée $(81 \%)$, plus de la moitié $(57 \%)$ se retrouvant dans des conditions particulièrement précaires du fait de leur appartenance à des ménages eux-mêmes démunis ${ }^{17}$.

Aussi, dans un second temps, pour essayer de retrouver des groupes d'individus démunis plus homogènes du point de vue des caractéristiques socio-économiques et du pouvoir de décision au sein du ménage, une typologie de cette population à faibles ressources a été effectuée en fonction de déterminants socio-économiques (genre, âge, position au sein du ménage, niveau d'étude, activité économique) ${ }^{18}$. Les groupes principaux sont ceux des garçons scolaires ou étudiants et des ménagères, qui représentent à eux deux $45 \%$ des citadins démunis (voir annexe). Les individus économiquement actifs se trouvent dans deux groupes, les jeunes actifs et les chefs de ménage, les deux derniers groupes étant constitués de jeunes filles, les unes inactives, les autres scolaires. Ces différents groupes d'individus démunis présentent des revenus individuels très faibles, de 3000 à 30000 FCFA mensuels, contre 85000 FCFA en moyenne pour les individus aisés. Avant de présenter les pratiques de mobilité de ces six groupes, nous explicitons l'influence du niveau de ressources du ménage sur les situations et les comportements individuels.

\footnotetext{
${ }^{17}$ Rappelons qu'il s'agit bien d'une appréhension volontairement très large des situations individuelles de manque de moyens et non pas du repérage des citadins pauvres. L'Enquête Prioritaire réalisée au Burkina Faso en 1994-95 conduit d'ailleurs à un taux d'incidence de la pauvreté de 7,4\% en milieu urbain (Lachaud, 1998).

18 Une analyse factorielle des correspondances multiples sur ces variables socio-démographiques permet de dégager trois axes principaux. A partir des coordonnées des individus sur ces trois axes, une classification ascendante hiérarchique, affinée par des classifications par nuées dynamiques, a débouché sur l'identification de six groupes.
} 


\section{NIVEAUX DE RESSOURCES, CARACTERISTIQUES SOCIALES ET MOBILITE}

$70 \%$ des individus démunis habitent dans des logements en banco ou en semi-dur, mais l'aisance du chef de ménage se traduit logiquement par une nette amélioration des conditions de logement. Ainsi, dans les ménages démunis, un individu démuni sur deux réside dans une maison traditionnelle en banco alors que ce n'est le cas que d'un sur dix dans les ménages plus favorisés. On vérifie aussi que les plus pauvres sont relativement concentrés dans les quartiers d'habitat dit « spontanés » situés en grande périphérie (tableau 3).

Environ trois individus démunis sur cinq sont célibataires, trois sur quatre ont moins de 35 ans, mais c'est dans les ménages aisés qu'on trouve le plus de jeunes $(88 \%$ des individus démunis ont moins de 35 ans, contre $67 \%$ dans les ménages démunis). Dans les ménages démunis, les enfants du chef sont plus nombreux que les autres dépendants (respectivement $37 \%$ et $31 \%$ ), ces proportions s'inversant lorsque le niveau de vie du ménage augmente. L'hypothèse d'un accueil plus fréquent d'autres parents dans les ménages aisés se trouve confirmée. Elle reflète les solidarités familiales mises en oeuvre pour faire face à la crise, solidarités dont les contraintes «[...] s'imposent aux plus forts économiquement en raison du système des droits et obligations $»^{19}$.

Tableau 3 : Lieu de résidence des différentes catégories d'individus (\%)

\begin{tabular}{lcccc}
\hline & Centre & $\begin{array}{c}\text { 1ère } \\
\text { couronne }\end{array}$ & $\begin{array}{c}\text { 2ème } \\
\text { couronne }\end{array}$ & Ensemble \\
\hline Individus démunis de ménages démunis & 10 & 37 & 53 & 100 \\
Individus démunis de ménages aisés & 14 & 50 & 36 & 100 \\
\hline Individus aisés dans des ménages démunis & $16^{*}$ & 65 & $19^{*}$ & 100 \\
Individus aisés dans des ménages aisés & 11 & 47 & 42 & 100 \\
\hline Ensemble & 12 & 44 & 44 & 100 \\
\hline
\end{tabular}

* effectifs inférieurs à 30 individus

L'inactivité économique prédomine parmi les individus à faibles ressources. Etudiants, inactifs, chômeurs et retraités constituent $71 \%$ des citadins démunis à Ouagadougou. La part restante est constituée d'actifs travaillant généralement dans le secteur informel. Le taux d'analphabétisme est élevé, de l'ordre de $29 \%$, et fort logiquement, plus encore lorsque le

19 Vimard, 1997, p. 154. 
ménage a lui-même de faibles ressources (37\%). L'enquête permet aussi de vérifier qu'une situation plus aisée du chef de ménage favorise la scolarisation des enfants et l'accueil de dépendants également scolarisés ${ }^{20}$. Ainsi, dans les ménages aisés, $50 \%$ des enfants et dépendants démunis sont étudiants et $16 \%$ seulement sont économiquement actifs, ces taux se situant respectivement à 25 et $40 \%$ lorsque le ménage dispose de faibles ressources.

Comme le montre le tableau 4, un tiers des individus à faibles ressources dispose de façon permanente d'un véhicule ( $21 \%$ ont un deux-roues moteur et $14 \%$ un vélo), $23 \%$ peuvent en utiliser occasionnellement un et $42 \%$ n'y ont jamais accès. L'aisance du ménage n'a qu'une faible influence sur la disponibilité individuelle d'un véhicule. Cette possibilité dépend avant tout de la capacité de l'individu à en financer lui-même l'achat et l'usage : $83 \%$ des individus aisés ont accès de façon permanente à un deux-roues moteur ou à une voiture.

Tableau 4 : Disponibilité des véhicules selon les ressources des individus et de leur ménage $(\%)$

\begin{tabular}{lccc}
\hline & $\begin{array}{c}\text { Individu démuni, } \\
\text { ménage démuni }\end{array}$ & $\begin{array}{c}\text { Individu démuni, } \\
\text { ménage aisé }\end{array}$ & Individu aisé \\
\hline Aucun mode de transport & 43,1 & 41,5 & 8,4 \\
Bicyclette, occasionnellement & 6,7 & 4,4 & 1,2 \\
Bicyclette, de façon permanente & 14,1 & 12,4 & 2,0 \\
Deux-roues moteur, occasionnellement & 15,0 & 20,3 & 5,0 \\
Deux-roues moteur, de façon permanente & 20,3 & 21,0 & 66,1 \\
Voiture, de façon permanente & 0,8 & 0,4 & 17,3 \\
\hline Ensemble & 100,0 & 100,0 & 100,0 \\
\hline
\end{tabular}

Une situation économique plus favorable conduit à une augmentation de la mobilité, et en particulier de la mobilité mécanisée, qui fait plus que doubler entre individus démunis et aisés (tableau 5). Mais plus encore que ces différences somme toute prévisibles, l'information essentielle est apportée par la distinction et le croisement des niveaux de ressources de l'individu et de son ménage. Quel que soit le revenu du chef de ménage, les individus à faibles ressources se déplacent moins pour le travail, parcourent des distances plus courtes et se déplacent plus souvent à pied, consacrant en moyenne 2500 FCFA mensuels à leur transport, soit le tiers de leur revenu propre.

${ }^{20}$ Lachaud, 1995. 
La situation économique individuelle est donc le facteur déterminant des conditions de déplacement. Il est vrai que les ménages classés comme aisés selon nos critères constituent près de la moitié des foyers et représentent une catégorie encore assez hétérogène. L'amélioration conséquente de la situation individuelle des personnes dépendant du chef de famille ne se produit peut-être qu'au sein de la minorité des ménages les plus aisés. Quoi qu'il en soit, le fait, pour une personne démunie, de vivre dans un ménage aisé a généralement peu de conséquence sur ses conditions de déplacement, de même qu'il améliore assez peu l'accès personnel aux moyens de transport du ménage.

Parmi les individus démunis, les différences de mobilité s'expliquent alors essentiellement par la diversité des statuts socio-économiques, le genre, la position dans le cycle de vie, l'activité, influençant le niveau et les formes de la mobilité urbaine.

Tableau 5 : Indicateurs de mobilité selon les ressources des individus et de leur ménage (en nombre moyen de déplacements quotidiens et \%)

\begin{tabular}{lccc}
\hline & $\begin{array}{c}\text { Mobilité } \\
\text { tous modes }\end{array}$ & $\begin{array}{c}\text { Mobilité } \\
\text { mécanisée }\end{array}$ & \% à pied \\
\hline Individus démunis dans des ménages démunis & 3,5 & 1,7 & 51,5 \\
Individus démunis dans des ménages aisés & 3,9 & 2,0 & 48,6 \\
\hline Individus aisés dans des ménages démunis & 4,1 & 3,7 & 12,3 \\
Individus aisés dans des ménages aisés & 4,7 & 4,3 & 9,9 \\
\hline Ensemble & 3,8 & 2,2 & 41,5 \\
\hline
\end{tabular}

\section{LES MOBILITES QUOTIDIENNES DES CITADINS DEMUNIS}

Parmi les six groupes d'individus démunis, les moins mobiles sont les ménagères, suivies des jeunes inactives, des chefs de ménage, des filles scolaires, des jeunes actifs et enfin, des garçons scolarisés. Le nombre de déplacements s'échelonne de 2,6 à 4,8 soit, pour les plus mobiles, des niveaux de mobilité similaires à la moyenne des individus aisés. Mais plus encore que les niveaux, ce sont les formes prises par cette mobilité qui laissent apparaître les écarts les plus significatifs.

Le motif de déplacement est le premier élément de différenciation des groupes d'individus à faibles ressources, compte tenu de statuts et de rôles économiques et familiaux très variés (graphique 1). 


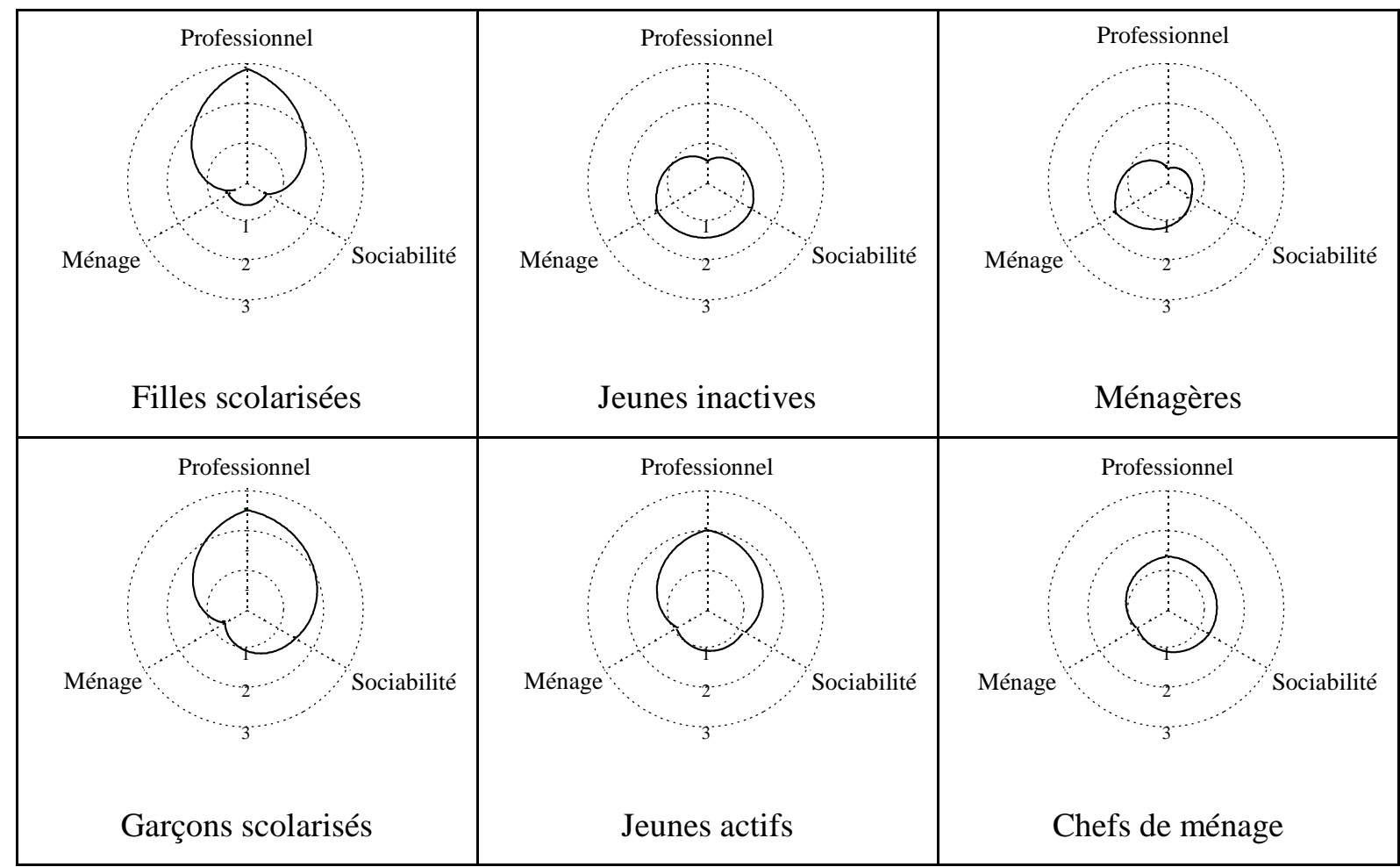

* «Ménage » regroupe les motifs achats (majoritaires), démarches, religion, approvisionnement en eau ou autre; "Professionnel» regroupe les déplacements liés au travail ou aux études; "Sociabilité » regroupe les déplacements motivés par des visites aux parents ou aux amis (très majoritaires), par les visites aux voisins ou par des activités de loisir.

Les scolaires, filles comme garçons, sont peu concernés par les activités extérieures liées à l'entretien du ménage et leur mobilité est fortement structurée par les études. Cela est particulièrement net dans le cas des jeunes filles scolarisées, pour qui la vie sociale en dehors du domicile est très limitée. Les achats constituent le premier motif de déplacement pour les ménagères et les jeunes inactives. Ces dernières se distinguent des autres groupes féminins par une vie sociale extérieure très développée, comparable même à celle des groupes masculins. La structure des motifs de déplacement des jeunes actifs est similaire à celle des garçons scolarisés, bien que moins structurée par l'occupation principale. Enfin, malgré un faible niveau de mobilité, les chefs de ménage présentent la structure de motifs la plus équilibrée.

Bien évidemment, par rapport aux individus aisés, tous ces groupes utilisent moins la voiture et les deux-roues moteur mais beaucoup plus la marche à pied, et un peu plus la bicyclette (graphique 2). Seuls les chefs de ménage recourent majoritairement aux deux-roues moteur, la moitié d'entre eux en disposant de façon permanente. De façon plus générale, parmi les 
citadins démunis, vélomoteurs et motos demeurent des modes de transport essentiellement masculins, du fait en premier lieu de statuts d'occupation distincts (les actifs et les scolaires, forts utilisateurs, sont majoritairement des hommes), mais aussi du fait de différences de rôles à statut identique ou proche, différences dont le meilleur exemple est donné par la comparaison des scolaires masculins et féminins.

Graphique 2: Nombre de déplacements par mode de transport pour les groupes d'individus démunis

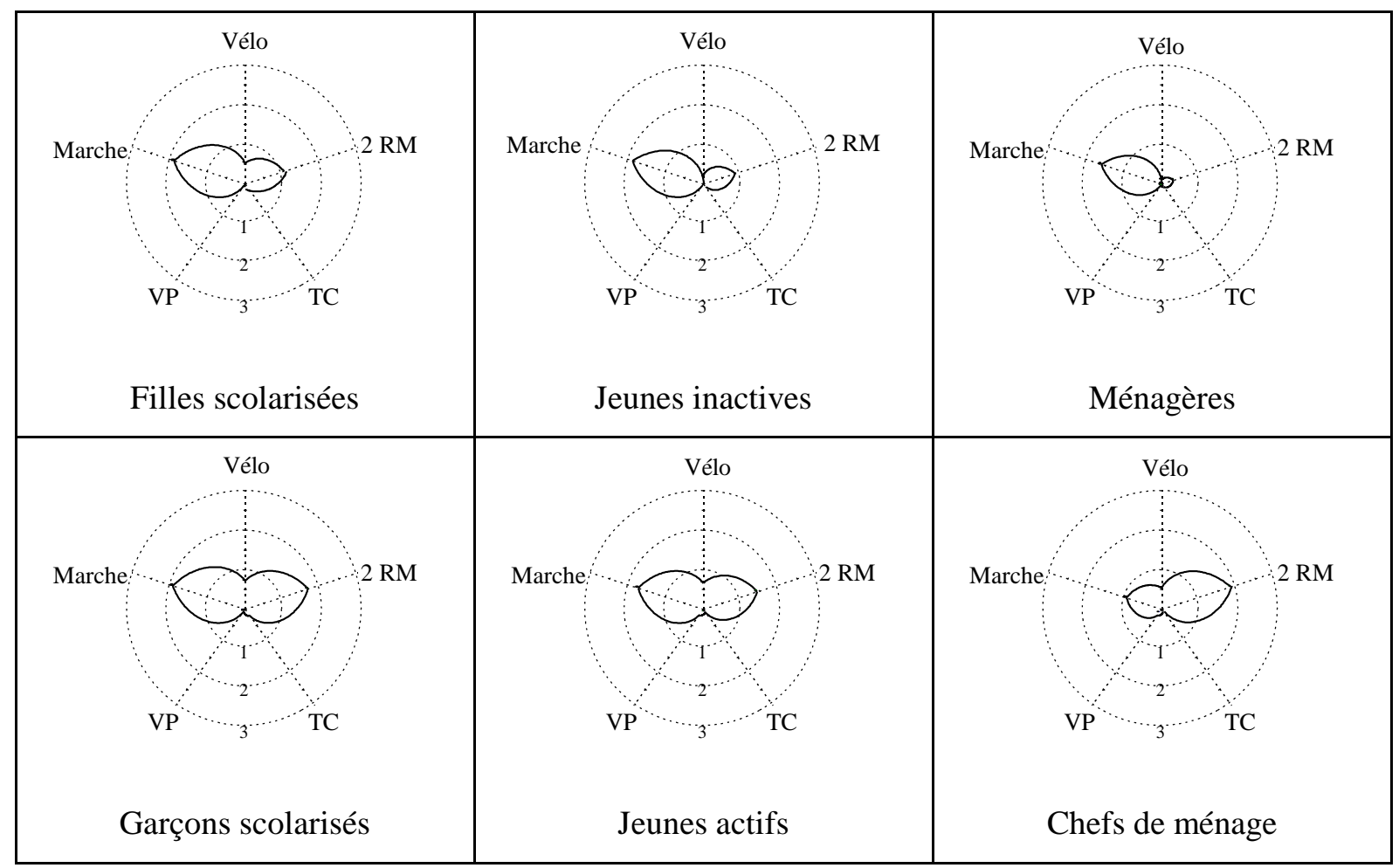

«2RM»: Deux roues-moteur (conducteur ou passager) ; «TC»: Transports Collectifs ;

«V » : Voiture Particulière (conducteur ou passager).

Pour les différents groupes d'individus démunis, le fait d'appartenir à un ménage aisé n'améliore pas fondamentalement les conditions de déplacement, comme le confirment les niveaux de mobilité : très légère hausse de la mobilité totale ( 0,1 à 0,2 déplacement), accroissement limité de la mobilité mécanisée (0,4 à 0,5 déplacement). Dans la répartition modale, la marche perd de 12 à 14 points selon les groupes mais demeure le premier mode de déplacement. Seule exception, sur laquelle nous reviendrons plus loin, les jeunes actifs voient au contraire leur mobilité baisser de 0,7 déplacement (la part de la marche à pied passant de $36 \%$ à $55 \%$ ). Ces variations dans l'usage des modes sont très liées au pourcentage 
d'individus ayant un accès permanent à un véhicule individuel, notamment un deux-roues moteur (tableau 6).

Tableau 6: Taux de disponibilité permanente d'un véhicule dans les groupes d'individus démunis selon le niveau de ressources du ménage (\% du total du groupe)

\begin{tabular}{l|cccccc}
\hline Ménage... & $\begin{array}{c}\text { Chefs de } \\
\text { ménage }\end{array}$ & $\begin{array}{c}\text { Garçons } \\
\text { scolarisés }\end{array}$ & $\begin{array}{c}\text { Filles } \\
\text { scolarisées }\end{array}$ & $\begin{array}{c}\text { Jeunes } \\
\text { inactives }\end{array}$ & Ménagères & $\begin{array}{c}\text { Jeunes } \\
\text { actifs }\end{array}$ \\
\cline { 2 - 7 } ...démuni & 76 & 38 & 28 & 13 & 10 & 45 \\
...aisé & sans objet & 50 & 38 & 20 & 16 & 36 \\
Différence & sans objet & +12 & +10 & +7 & +6 & -9 \\
\hline
\end{tabular}

Ces pourcentages et leurs variations permettent de faire ressortir les positions relatives des différents membres du ménage et les arbitrages faits autour d'un bien rare, le véhicule individuel. Les chefs de ménage sont de loin les plus favorisés, suivis par les deux groupes de scolaires. Cependant, les garçons scolarisés se trouvent dans une meilleure position que leurs homologues féminins, la scolarisation des garçons étant perçue comme un investissement bien plus rentable que celle des filles ${ }^{21}$. On le voit, leur situation relativement privilégiée se traduit non seulement par des taux de scolarisation plus élevés et une durée de scolarisation plus longue, mais aussi par de meilleures conditions de déplacement entre le domicile et l'école.

$\mathrm{Au}$ sein des ménages (et plus encore lorsque ceux-ci disposent de faibles ressources), deux groupes féminins, les jeunes inactives et les ménagères, paraissent presque systématiquement tenus à l'écart de l'attribution des moyens de transport. L'arbitrage apparaît clairement : leur place étant le foyer, pourquoi leur permettre l'accès à un véhicule qui est considéré comme plus utile par ailleurs, puisqu'il facilite la réalisation d'une activité actuellement (pour les actifs) ou potentiellement (pour les jeunes scolarisés) productive?

Enfin, la situation des jeunes actifs à faibles ressources n'est paradoxale qu'en apparence. Leur accès à un véhicule individuel, plus fréquent dans les ménages démunis que dans les ménages aisés, traduit là encore une hiérarchisation implicite des besoins au sein des ménages. Vient à l'appui de cette interprétation, le fait que, lorsque le ménage compte plusieurs actifs, l'accès permanent d'un jeune actif à un deux-roues est moins fréquent au sein des ménages aisés que chez les ménages démunis. Dans les premiers, pour l'attribution du ou des véhicules,

21 Marcoux, 1995. 
un actif aux faibles revenus apparaît sans doute moins prioritaire que le chef de ménage et/ou d'autres actifs mieux rémunérés que lui.

On le voit, dans des situations fortement contraintes par la faiblesse des ressources individuelles, l'attribution des moyens de transport est étroitement liée aux différences de statut au sein du ménage.

\section{DE LA MOBILITE QUOTIDIENNE AUX POLITIQUES DE TRANSPORT URBAIN}

Ces résultats permettent d'éclairer certains aspects de la relation «pauvreté-difficultés dans les déplacements urbains ", souvent évoquée mais encore trop peu analysée à l'aune de données quantitatives. Les spécificités mises en évidence montrent d'une part la grande hétérogénéité des populations à faibles ressources et la grande diversité de leurs besoins dans le domaine de la mobilité quotidienne, d'autre part l'affectation sélective des moyens de transport au sein des ménages. Elles valident notre parti pris de départ dans la catégorisation des situations individuelles. Il est vrai que la domination des deux-roues dans le système de transport à Ouagadougou, qui offre des possibilités de déplacement très différenciées aux individus mais à un coût élevé, rend ces résultats particulièrement nets en renforçant l'importance des arbitrages au sein des ménages. Il serait ainsi intéressant de tester leur degré de validité dans des contextes urbains où les transports collectifs tiennent une place plus importante.

L'hétérogénéité sociale existant au sein des populations démunies est bien entendu liée à des statuts sociaux très divers, selon le genre, l'âge, la situation professionnelle et la position dans le ménage, qui nous ont permis de mettre en évidence six groupes aux pratiques de déplacements contrastées. Ainsi, par exemple, la mobilité quotidienne des ménagères, sans revenus propres et pratiquement sans accès à un véhicule individuel, a peu de points communs avec celle des jeunes actifs, célibataires, qui doivent se déplacer pour le travail et disposent presque librement de leurs revenus.

En matière d'accès aux véhicules, les arbitrages familiaux tiennent compte des besoins de déplacement présents et «objectifs », mais s'effectuent aussi, implicitement, sur la base d'un calcul économique dont les bénéfices seront recueillis dans l'avenir. Ils s'exercent alors préférentiellement vers les individus considérés comme actuellement ou potentiellement « utiles », c'est-à-dire les actifs et les scolaires (et, parmi ces derniers, les garçons plutôt que les filles), tandis que les femmes au foyer, épouses, filles ou dépendantes inactives, restent à 
l'écart de l'affectation des véhicules au sein du ménage. Le rôle socialement défini de la femme au foyer s'organise autour des tâches ménagères et des soins aux enfants, le rare temps libre étant passé avec des voisines dans la même situation. Dans ce cadre, avoir un mode de transport individuel mécanisé à sa disposition apparaît «rationnellement» superflu. Par contre, les membres du ménage économiquement actifs et les scolaires doivent aller travailler ou assister aux cours. Pour eux, l'utilisation d'un deux-roues moteur (ou, à défaut, d'un vélo) semble plus appropriée que la marche à pied. De fait, la prise en compte de la position sociale et des obligations de chacun amène à restreindre l'accès de certaines catégories démunies à un mode de transport plus efficace que la marche, et finalement, à pérenniser les inégalités existantes.

Ces résultats ne sont pas sans conséquences en matière de lutte contre la pauvreté dans les villes africaines. Compte tenu de l'étendue et de la diversité des besoins des groupes défavorisés, il n'est pas possible de définir et de mettre en oeuvre des politiques sectorielles bénéficiant de manière équivalente à tous. Dans un contexte de rareté des ressources disponibles, il revient aux pouvoirs publics la responsabilité de fixer clairement des objectifs prioritaires et d'arbitrer entre les besoins des différents groupes de citadins. Les mesures visant à développer ou à rendre plus abordables les transports en commun ou le transport individuel économique (notamment la bicyclette) peuvent à court terme diminuer aussi bien les dépenses que la pénibilité et le temps passé quotidiennement en transport des actifs à bas revenus et des scolaires. En revanche, les politiques de transport ne semblent pas actuellement en mesure d'offrir une réelle opportunité aux femmes inactives d'élargir leur espace de vie quotidien. Pour ces femmes, ménagères ou jeunes sans emploi, les tâches domestiques (éducation des enfants, approvisionnement en eau ou en bois de feu, pilage du mil...) laissent peu de place à d'autres formes de participation sociale, à travers le système éducatif ou par une activité rémunérée par exemple. Ces rythmes de vie traditionnels, très astreignants, sont caractéristiques notamment des «villages » et des quartiers spontanés de la périphérie des capitales africaines. Leur évolution passe d'abord très probablement dans un premier temps par une amélioration de l'accès aux principaux services urbains. Plus qu'un accroissement de l'offre de transport, une stratégie soutenue d'équipement des quartiers périphériques paraît réellement à même de prendre en compte les besoins de ces groupes souvent implicitement laissés pour compte dans les politiques de transports urbains.

\section{Bibliographie}


Banque Mondiale (1990), Rapport sur le développement dans le monde 1990. La pauvreté, Washington.

Diaz Olvera L., Plat D. (1994), La mobilité des individus, in X. Godard (sous la dir. de), Analyse du système de déplacements à Ouagadougou, Ouagadougou-Arcueil-Lyon, CEDRESINRETS-LET.

Diaz Olvera L., Plat D. (1997), Confisquée, partagée, consensuelle. La voiture à Ouagadougou, in O. Figueroa, X. Godard, E. Henry (éditeurs), Mobilité et politiques de transport dans les villes en développement, Arcueil, INRETS, actes $\mathrm{n}^{\circ} 55$.

Diaz Olvera L., Plat D., Pochet P. (1996), Mobilités urbaines comparées en Afrique de l'Ouest : Bamako et Ouagadougou, Selected Proceedings of the 7th WCTR Conference, Oxford, Elsevier Science.

Gannon C.A., Liu Z. (1997), Poverty and transport, Discussion paper, TwU Papers, n³0, Washington, The World Bank.

Godard X., Teurnier P. (1992), Les transports urbains en Afrique à l'heure de l'ajustement. Redéfinir le service public, Paris-Arcueil, Karthala-INRETS.

Godard X. (1997), Mobilité et pauvreté, enseignements de l'expérience ouest-africaine, Forum international Habitat sur la Pauvreté Urbaine, Florence, 9-13 novembre.

Grieco M., Apt A., Turner J. (1996), At Christmas and on rainy days. Transport, travel and the female traders of Accra, Avebury, Aldershot.

Jaglin S. (1995), Gestion partagée à Ouagadougou. Pouvoirs et périphéries (1983-1991), Paris, Karthala-ORSTOM.

Jaglin S., Le Bris E., Marie A., Osmont A., Ouattara A., Ouedraogo J.-P., Piron-Ayraud M., Poussi M., Sanou B. (1992), Les enjeux des extensions urbaines à Ouagadougou (Burkina Faso), Rapport de fin d'étude pour le Ministère de la recherche et de la technologie, $\mathrm{n}^{\circ} 48$, CNRST du Burkina Faso, IRSSH, ORSTOM, DGUT du Burkina Faso, Université de Paris VIII, Institut Français d'Urbanisme.

Lachaud J.-P. (1995), Marché du travail et exclusion sociale dans les capitales d'Afrique francophone : quelques éléments d'analyse, Revue Tiers-Monde, t. XXXVI, nº142.

Lachaud J.-P. (1998), Modélisation des déterminants de la pauvreté et marché du travail en Afrique : le cas du Burkina Faso, Document de travail n 32, Bordeaux, Centre d'Economie du Développement.

Marcoux R. (1995), Fréquentation scolaire et structure démographique des ménages en milieu urbain au Mali, Cahiers des Sciences Humaines, vol. 31, n³. 
Marie A. (1995), «Y a pas l'argent » : l'endetté insolvable et le créancier floué, deux figures complémentaires de la pauvreté abidjanaise, Revue Tiers Monde, t. XXXVI, $\mathrm{n}^{\circ} 142$.

Marie A. (éditeur, 1997), L'Afrique des individus, Paris, Karthala.

Ngabmen H. (1997), Crise des transports collectifs urbains et stratégies d'adaptation : le cas de Yaoundé, in O. Figueroa, X. Godard, E. Henry (éditeurs), Mobilité et politiques de transport dans les villes en développement, Arcueil, INRETS, actes $\mathrm{n}^{\circ} 55$.

Pochet P., Klein O., Toilier F., Godard X., Malou N., Ballo A., Coulibaly M., Djenapo M., Keita M. (1995), Les transports urbains non motorisés en Afrique sub-saharienne. Le cas du Mali, Vaulx-en-Velin-Arcueil-Bamako, LET-INRETS-ARETRANS.

Salama P., Valier J. (1995), Mesures des pauvretés et de l'appauvrissement, Revue Tiers Monde, t. XXXVI, $\mathrm{n}^{\circ} 142$.

Sethi K., Bhandari A. (1996), Impact of Planned Improvements in Public Transport on the Urban Poor in Lagos, Actes de la CODATU VII. Tome 1, New Delhi, 12-16 février.

Vimard P. (1997), Modernisation et crise de la famille (Afrique sub-saharienne), Autrepart, $\mathrm{n}^{\circ} 2$.

Vuarin R. (1997), Sur le processus d'exclusion des solidarités protectrices à Bamako (Mali), in ORSTOM, CEDERS, Atelier ménages et crise, Marseille, 24-26 mars.

Werner J.-F. (1997), Itinéraires individuels à la marge. Etudes de cas sénégalais, in A. Marie (éditeur), L'Afrique des individus, Paris, Karthala. 


\section{Annexe : Composition des six groupes d'individus démunis}

\begin{tabular}{ll}
\hline Ménagères & $99 \%$ de femmes \\
$\mathbf{2 2} \%$ & $83 \%$ d'épouses \\
RI : 3700 & $42 \%$ ont entre 19-34 ans et $49 \%$ ont de 35 à 54 ans \\
RI (>0) : 15000 & $75 \%$ sont analphabètes \\
& $71 \%$ sont inactives, $26 \%$ sont non salariées \\
\hline Jeunes actifs & $96 \%$ d'hommes \\
$\mathbf{1 3} \%$ & $52 \%$ de dépendants et $41 \%$ d'enfants \\
RI : 14700 & $72 \%$ ont entre 19 et 34 ans \\
RI $(>0): 23100$ & $39 \%$ ont fait des études primaires et $26 \%$ sont analphabètes \\
& $67 \%$ travaillent dans le secteur informel
\end{tabular}

Chefs de ménage $\quad 93 \%$ d'hommes

$11 \% 98 \%$ de chefs de ménage

RI : $30300 \quad 61 \%$ ont de 35 à 54 ans et $30 \%$ ont 55 ans ou plus

RI (>0) : $39600 \quad 31 \%$ ont fait des études primaires et $51 \%$ sont analphabètes

$51 \%$ travaillent dans le secteur informel et $23 \%$ sont salariés

\begin{tabular}{ll}
\hline Jeunes inactives & $70 \%$ de femmes \\
$\mathbf{1 6} \%$ & $59 \%$ de dépendants et $31 \%$ d'enfants \\
RI : 2900 & $61 \%$ ont entre 19 et 34 ans et $38 \%$ entre 14 et 18 ans \\
RI (>0) : 16700 & $43 \%$ ont fait des études primaires, 30\% des études secondaires \\
& $76 \%$ sont inactives ou au chômage \\
\hline Filles scolarisées & $100 \%$ de femmes \\
16 \% & $100 \%$ de scolaires ou d'étudiantes \\
RI : 3500 & $70 \%$ de filles du chef, $30 \%$ de dépendantes \\
RI (>0) : 17700 & $59 \%$ ont entre 14 et 16 ans et $41 \%$ entre 19 et 34 ans \\
& $8 \%$ sont en études supérieures, $86 \%$ dans le secondaire \\
\hline Garçons scolarisés & $100 \%$ d'hommes \\
23 \% & $90 \%$ de scolaires ou d'étudiants \\
RI : 5300 & $71 \%$ de fils du chef, $29 \%$ de dépendants \\
RI (>0) : 22700 & $48 \%$ ont entre 14 et 18 ans, $52 \%$ entre 19 et 34 ans \\
& $15 \%$ sont en études supérieures, $79 \%$ dans secondaire \\
\hline
\end{tabular}

* Les pourcentages en caractères gras sont calculés par rapport à l'échantillon de l'ensemble des pauvres. Les autres pourcentages font référence au total du groupe.

$R I$ : Revenu individuel moyen du groupe ;

$R I(>0):$ Revenu individuel moyen pour les personnes du groupe déclarant un revenu non nul. 